\title{
MANEJO LAPAROSCÓPICO DE DISPOSITIVO INTRAUTERINO INCRUSTADO EN PARED VESICAL CON LITIASIS VESICAL SECUNDARIA
}

\author{
Jaime Zarhi T. ' , Fernando Vacca G. ${ }^{1}$, Pablo Sanhueza R. ${ }^{1}$, Jorge Díaz M. ${ }^{2}$, \\ Rodrigo Riffo R. ${ }^{2}$ \\ ${ }^{1}$ Servicio de Obstetricia y Ginecología, ${ }^{2}$ Servicio de Urología, Hospital El Pino.
}

\section{RESUMEN}

Se presenta el caso clínico de la migración intravesical de dispositivo intrauterino con litiasis vesical secundaria. El diagnóstico se realizó 20 años después de la inserción del dispositivo, por la aparición de síntomas urinarios bajos a repetición y hematuria. La ultrasonografía y la radiografía de pelvis son herramientas diagnósticas relevantes en la sospecha inicial de esta patología, que debe siempre completarse con tomografía pélvica y cistoscopia.

\section{PALABRAS CLAVE: Dispositivo intrauterino, migración intravesical, litiasis vesical}

\section{SUMMARY}

This is a case report of an intravesical migration of an intrauterine device, with secondary vesical lithiasis. The diagnosis was made 20 years after the insertion of the device, because of the appearance of recurrent low urinary tract symptoms and haematuria. Ultrasonography and pelvic radiography are important diagnostic tools on the initial study of this pathology that must be completed with pelvic tomography and cistoscopy.

KEY WORDS: Intrauterine device, intravesical migration, vesical lithiasis

\section{INTRODUCCIÓN}

Los dispositivos intrauterinos son un método anticonceptivo reversible ampliamente usado, altamente efectivo y seguro en el control de la fertilidad. Ocasionalmente entre un 0,87 a 1,6 por 1000 puede asociarse a perforación uterina y su expulsión dentro de la cavidad abdominal o retroperitoneal $(1,2)$. Esta complicación ocurre más frecuentemente en el momento de la inserción, pero esta puede también ocurrir tardíamente, siendo la mayoría de las veces asintomática, por lo que la paciente puede permanecer meses o años antes que se realice el diagnóstico de perforación (3). El tratamiento más aceptado de esta complicación, es la remoción quirúrgica ya sea por laparotomía o laparoscopia, por el riesgo que significa la formación de adherencias e injuria de órganos adyacentes como epiplón, rectosigmoide, vejiga, apéndice y órganos retroperitoneales $(1,2,4)$.

A pesar de la relación anatómica con la vejiga, la migración transuterina transvesical del DIU es una rara y poco común complicación, correspondiendo solo al $2 \%$ de las localizaciones abdomino-pélvicas. 
A diferencia de otras localizaciones, su ubicación intravesical lo hace actuar como cuerpo extraño, lo que produce precipitación de calcio con formación secundaria de un cálculo vesical $(3,5)$. Los cálculos vesicales primarios son muy raros, salvo en países como China, India e Indonesia donde el déficit de proteínas y fosfatos es importante. Los cálculos vesicales secundarios se dan un $95 \%$ en varones y generalmente en pacientes con uropatía obstructiva o vejiga neurogénica, siendo un fenómeno relevante en la fisiología de la formación del cálculo el elevado residuo urinario y la infección (6).

Ocasionalmente un cálculo vesical puede ser secundario a un cuerpo extraño, como las mallas de TOT y TVT en las cirugías de corrección de incontinencias de orina, o menos frecuentemente a la migración de un dispositivo intrauterino perforado a vejiga. Su sintomatología puede incluir todos los síntomas del tracto urinario inferior, ya sea irritativos u obstructivos, con o sin infección, retención urinaria, hematuria e incontinencia de orina, o presentarse asintomático como hallazgos en un examen de rutina $(6,7)$.

Se presenta el caso de litiasis vesical secundaria a un dispositivo intrauterino perforado e incrustado en pared vesical, diagnóstico realizado 22 años después de su inserción.

\section{Caso clínico}

Paciente de 49 años, multípara de 4 , con el antecedente de inserción de DIU tipo T Cu postparto en 1985, el cual no fue controlado. Inesperadamente, 3 años más tarde (1988) se embaraza siendo sometida a operación cesárea más esterilización en julio de 1990, donde el dispositivo no se recuperó durante la revisión y extracción de placenta, por lo que se presumió que lo había expulsado.

A fines del 2005, inicia disuria, urgencia miccional, hematuria intermitente y molestias hipogástricas acompañadas de pujo y tenesmo muy intensos, lo que motivo después de meses de evolución se solicitara una tomografía axial computarizada que mostró imagen compatible con cálculo que medía $50 \mathrm{~mm}$ de diámetro y alrededor de el, imagen ecorrefingente perforando pared posterior de vejiga que correspondería a DIU tipo T Cu (Figura 1). Con estos antecedentes la paciente es enviada a nuestro servicio para evaluación y completar estudio. Se completa estudio con ecotomografía trans- vaginal que no evidenció DIU en cavidad uterina y una radiografía de abdomen simple que lo mostró en posición invertida en cavidad pélvica y alrededor de una de sus ramas una imagen radio-opaca bien delimitada, que fue informada como probable cálculo vesical (Figura 2). Se realiza cistoscopia diagnóstica, que confirma el hallazgo de un cálculo vesical de $5 \mathrm{~cm}$ adherido a DIU que perfora la pared vesical por cara posterior (Figura 3), por lo que no es factible su extracción vía endoscópica. Se decide efectuar cistolitotomía previa instalación de catéteres ureterales.

Bajo anestesia general y en posición de litotomía se realiza neumoperitoneo con aguja de Veress en región umbilical. Se instalan 2 trocares de $10 \mathrm{~mm}$ a nivel umbilical y fosa iliaca derecha y 2 trocares de $5 \mathrm{~mm}$ suprapúbico y fosa iliaca izquierda. Se liberan adherencias y se rechaza intestino hacia cefálica. Llene vesical con $200 \mathrm{cc}$, identificando plano entre cara vesical posterior y útero. Se separan ambas estructuras identificándose DIU adherido firmemente a gran fibrosis perivesical. Cistotomía por cara posterior identifica litiasis de $5 \mathrm{~cm}$ adherida al DIU, se reseca fibrosis vesical y se extrae cálculo y DIU en bolsa por trocar de $10 \mathrm{~mm}$. Cistorrafia en 1 plano con vicril 2/0. Hemostasia conforme, sonda Foley 18 y drenaje al lecho. Se retira drenaje al 3er día y la paciente es dada de alta con sonda vesical la que se retira al $12^{\circ}$ día, sin incidentes.

\section{DISCUSIÓN}

Una de las mayores aunque infrecuentes complicaciones relacionadas con la inserción de un DIU es la perforación hacia la cavidad pélvica-abdominal. Su incidencia reportada es de 0,87 a 1,6 x 1000 inserciones, comprometiendo los órganos adyacentes como ligamento ancho, anexos, vejiga, epiplón y peritoneo, además de estructuras más distantes como apéndice y colon sigmoide $(1,2,7,8)$.

El mecanismo exacto de migración de un DIU o cuando exactamente ocurre la perforación hacia la cavidad pélvica-abdominal no esta claro, pero se cree que uno de los mecanismo es durante la inserción, produciéndose en forma directa la perforación hacia la cavidad; pero la migración hacia las estructuras adyacentes de un DIU parcialmente perforado en el miometrio, es una de las teorías reconocidas en pacientes en que el diagnóstico se realizó años después de la inserción inicial $(1,3,9)$. 


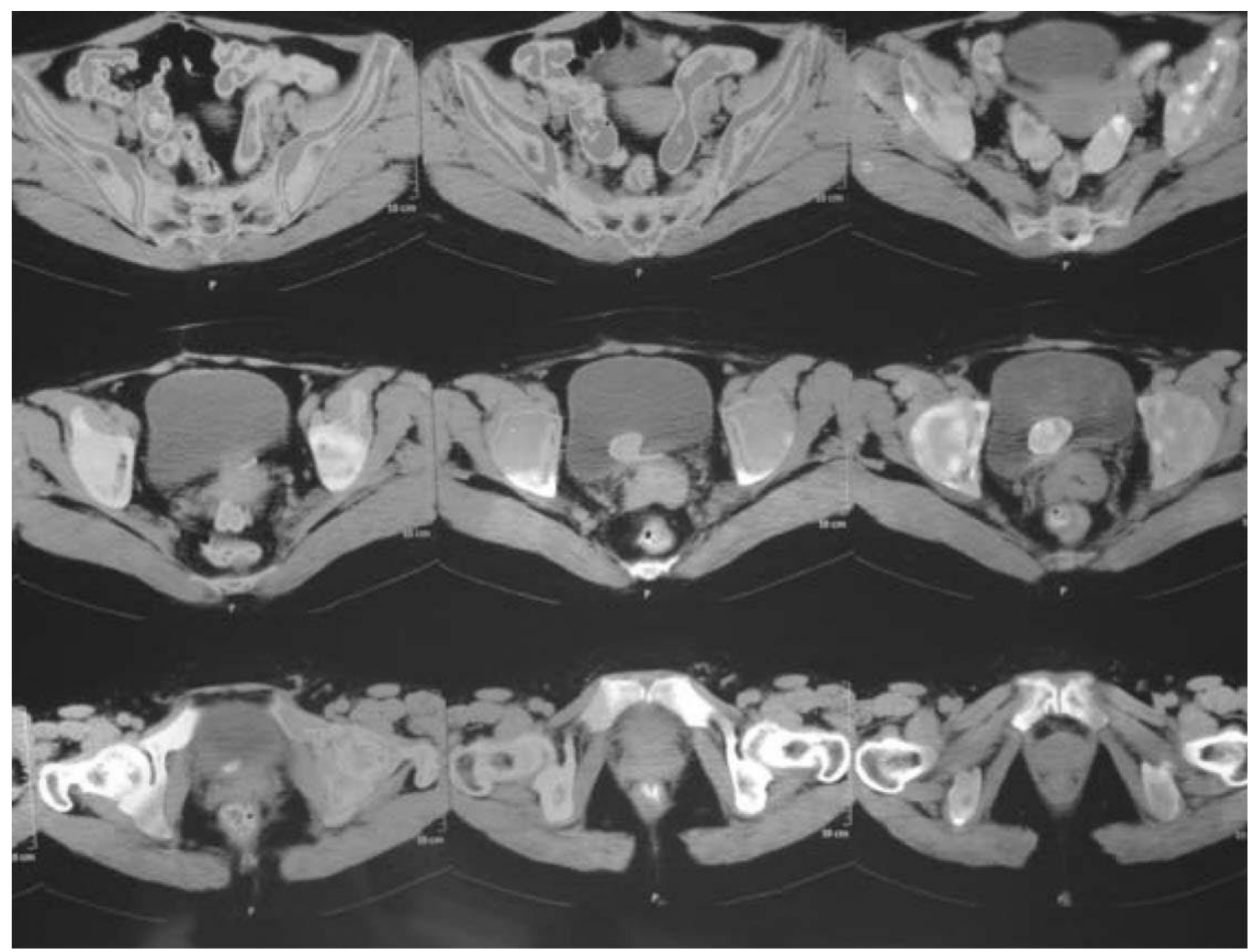

Figura 1. TAC pélvico que muestra DIU incrustado en pared vesical

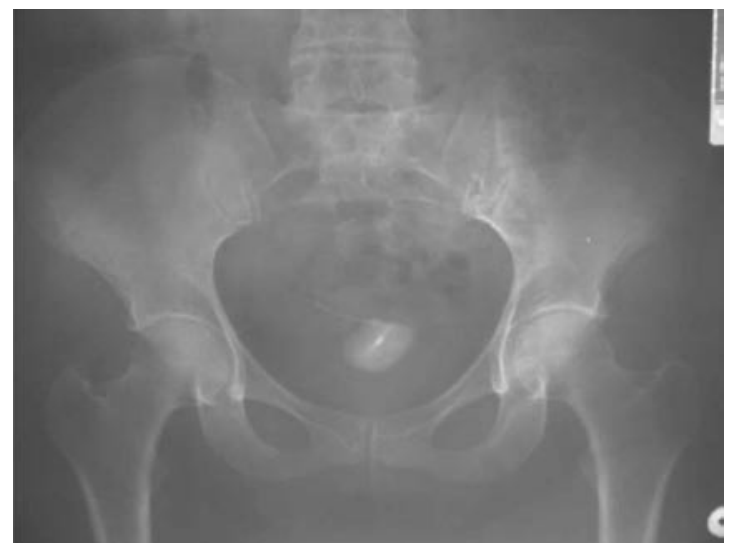

Figura 2. Radiografía de pelvis que muestra cálculo vesical alrededor de rama vertical del DIU.

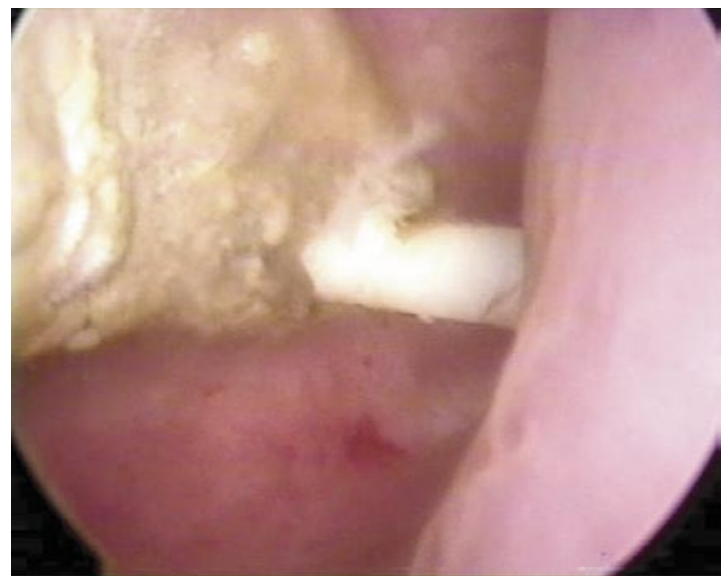

Figura 3. Cistoscopia que muestra rama de DIU incrustado en pared vesical con formación de cálculo. 
La migración de un DIU intravesical, ha sido raramente reportado en la literatura y puede tener lugar varios años antes que la sintomatología ocurra, ya que se trata generalmente de pacientes asintomáticas donde el diagnóstico se realiza como hallazgo al solicitar una ecotomografía por control ginecológico. En nuestro caso clínico el período de latencia entre la inserción del DIU y la aparición de los síntomas fue de 20 años, donde a raíz de síntomas urinarios bajos y hematuria, se realizó TAC abdomino-pélvico que mostró cálculo vesical con un DIU perforado en vejiga. Por lo tanto uno debería considerar la realización de una ecotomografía transvaginal inmediatamente después de la inserción, especialmente si esta resultó dificultosa o si la paciente se quejó que fue muy dolorosa (3). En la mayoría de los casos publicados en la literatura de DIU intravesicales, el diagnóstico se realizó como hallazgo al estudiar pacientes con variada sintomatología urinaria baja y no por sospecha primaria de migración del DIU (10). En una revisión de la literatura efectuada por Kassab y Audra, de un total de 165 DIU perforado solo 23 (14\%) correspondieron a localizaciones vesicales (11). Nosotros publicamos en la Revista Chilena de Obstetricia y Ginecología 18 DIU perforados a cavidad abdominal y no tuvimos ninguna localización intravesical (12).

Una vez que el DIU a migrado a vejiga, este actúa como cuerpo extraño, especialmente aquellos con componentes metálicos como la TCu, lo que puede causar precipitación de calcio y formación de cálculos en las vías urinarias en un plazo corto, dependiendo de la susceptibilidad del huésped y a la duración de la injuria, que es lo más probable que ocurrió en nuestro caso clínico $(5,13)$.

Aunque el examen vaginal y la ultrasonografía son herramientas fundamentales en la sospecha de un DIU perforado a abdomen, no es suficiente para su localización intravesical, por lo que es indispensable solicitar una Rx de pelvis, TAC y cistoscopia que no solo permite detectar si el dispositivo esta o no en la cavidad pélvica, sino además facilitar su ubicación durante el acto operatorio como fue en nuestro caso clínico, que nos permitió su ubicación intravesical concomitante con el cálculo vesical $(10,13,14)$.

Una vez hecho el diagnóstico, el tratamiento debe ser siempre quirúrgico y lo primero es realizar una cistoscopia, que además de confirmar el diagnóstico inicial, permite decidir por el tamaño del cálculo la factibilidad de fragmentación y extracción vía endoscópica, de lo contrario, en caso de cálculos mayores de $5 \mathrm{~cm}$ de diámetro la cistolitotomía ya sea por laparotomía o laparoscopia es el tratamiento mas adecuado $(3,6)$. En nuestro caso clínico, por tratarse de un cálculo de $5 \mathrm{~cm}$ adherido al DIU y que perforaba la pared vesical, realizamos una cistolitotomía vía laparoscópica que resultó muy laboriosa, ya que la rama vertical estaba incrustada en la pared vesical y la horizontal en el espesor del calculo que además estaba muy adherido a la pared vesical.

\section{CONCLUSIONES}

La presencia de un cálculo vesical primario, es una patología poco común, por lo tanto su presencia nos obliga a descartar un cuerpo extraño intravesical, que puede ser material de sutura no absorbible, una malla de TVT o TOT, o menos frecuente la migración intravaginal de un dispositivo intrauterino. La presencia de síntomas del tracto urinario bajo, hematuria, dolores hipogástricos e infecciones urinarias recurrentes nos obliga a realizar una anamnesis dirigida sobre el uso de dispositivo intrauterino y expulsiones no confirmadas, que debe incluir una cistoscopia, ecotomografía transvaginal y radiografía de pelvis. Los cálculos menores de $5 \mathrm{~cm}$ pueden ser removidos vía cistoscopia, previa fragmentación, en caso contrario el tratamiento debe ser la cistolitotomía por laparotomía o laparoscopia. La precocidad del diagnóstico de esta iatrogenia, mediante control ecográfico postinserción de DIU, facilita la extracción del cuerpo extraño y evita la aparición de complicaciones como litiasis, fístulas y embarazos no deseados.

\section{BIBLIOGRAFÍA}

1. Caliskan E, Oztürk N, Dilbaz BO, Dilbaz S. Analysis of risk factors associated with uterine perforation by intrauterine devices. Eur J Contracept Reprod Health Care 2003;8(3): 150-5.

2. Miranda L, Settembre A, Capasso P, et al. Laparoscopic removal of an intraperitoneal translocated intrauterine contraceptive device. Eur J Contracept Reprod Health Care 2003;8(2):122-5.

3. Khan ZA, Khan SA, Williams A, Mobb GE. Intravesical migration of levonorgestrel- releasing intrauterine system (LNG-IUS) with calculus formation. Eur J Contracept Reprod Health Care 2006;11(3):243-5.

4. Markovitch O, Klein Z, Gidoni Y, Holzinger M, Beyth Y. Extrauterine mislocated IUD: is surgical removal mandatory? Contraception 2002;66(2):105-8.

5. Ozçelik B, Serin IS, Basbug M, Aygen E, Ekmekçioglu O. Differential diagnosis of intra-uterine device migrating to bladder using radiographic image of calculus formation and review of literature. Eur J Obstet Gynecol Reprod Biol 2003;108(1):94-6.

6. Salazar A, Orellana N, Cortes M, Montigio C. Litiasis vesical secundaria a dispositivo intrauterino incrustado en pared vesical: revisión de 2 casos clínicos. Rev Chil Urol 2002;67(1):85-6. 
7. Singh I. Intravesical Cu-T emigration: an atypical and infrequent cause of vesical calculus. Int Urol Nephrol 2007;39(2):457-9.

8. Ozgür A, Sişmanoğlu A, Yazici C, Coşar E, Tezen D, Ilker $Y$. Intravesical stone formation on intrauterine contraceptive device. Int Urol Nephrol 2004;36(3):3458.

9. Dede FS, Dilbaz B, Sahin D, Dilbaz S. Vesical calculus formation around a migrated copper-T 380-A. Eur J Contracept Reprod Health Care 2006;11(1):50-2.

10. Nouira Y, Rakrouki S, Gargouri M, Fitouri Z, Horchani A. Intravesical migration of an intrauterine contraceptive device complicated by bladder stone: a report of six cases. Int Urogynecol J Pelvic Floor Dysfunct 2007;18(5):575-8.
11. Kassab B, Audra P. The migrating intrauterine device. Case report and review of the literatura. Contracept Fertil Sex 1999;27(10):696-700.

12. Zarhi J, Vacca F, Jarsun J, et al. DIU abdomino-pélvico: manejo laparoscópico. Rev Chil Obstet Ginecol 2005;70(6):369-74.

13. Insausti J, Urresola O, Ibañez S, Atilano S, Aguinaga A, Larrea B. Perforación utero-vesical secundaria a un dispositivo intrauterino con formación de litiasis vesical: a propósito de dos casos. Radiología 2007;49(2):129-32.

14. Istanbulluoglu MO, Ozcimen EE, Ozturk B, Uckuyu A, Cicek T, Gonen M. Bladder perforation related to intrauterine device. J Chin Med Assoc 2008;71(4):2079. 